INPLASY

PROTOCOL

To cite: Xu et al. Effectiveness and safety of moxibustion treatment for Polycystic Ovary

Syndrome: a protocol for systematic review and metaanalysis. Inplasy protocol 2020110049. doi:

10.37766/inplasy2020.11.0049

Received: 12 November 2020

Published: 12 November 2020

Corresponding author:

Kou Xu

1932707307@stmail.hbtcm.edu.cn

Author Affiliation:

Hubei University of Traditional

Chinese Medicine, Hubei

Provincial Hospital of Traditional

Chinese Medicine

Support: Research Project.

Review Stage at time of this submission: The review has not yet started.

Conflicts of interest:

None.

\section{Effectiveness and safety of moxibustion treatment for Polycystic Ovary Syndrome: a protocol for systematic review and meta-analysis}

Xu, K1; Zhou, Z2; Huang, W3; Lv, S4; Zhang, Y5; Wan, C6.

Review question / Objective: The study will evaluate the curative effect and adverse effects of moxibustion therapy for Polycystic Ovary Syndrome, in order to provide a reference for clinical treatment.

Condition being studied: Polycystic Ovary Syndrome (PCOS) is one of the most common endocrine diseases in women of adolescence and childbearing period. Its clinical manifestations include irregular menstruation, infertility, hyperandrogen signs, and polycystic ovary symptoms. Still, Patients with PCOS may also have metabolic abnormalities such as obesity, insulin resistance (IR), and dyslipidemia. PCOS is a high-risk factor for type 2 diabetes, cardiovascular and cerebrovascular diseases and endometrial cancer. At present, there is no effective cure for PCOS due to its unclear cause, therefore, symptomatic treatment has been the main treatment for a long time. Some recent trials indicate that moxibustion can be effective for PCOS. However, most of them are the combination of moxibustion and other therapies. To assess the effectiveness and safety of moxibustion treatment for PCOS, we designed this protocol for systematic review and meta-analysis.

INPLASY registration number: This protocol was registered with the International Platform of Registered Systematic Review and Meta-Analysis Protocols (INPLASY) on 12 November 2020 and was last updated on 12 November 2020 (registration number INPLASY2020110049).

\section{INTRODUCTION}

Review question / Objective: The study will evaluate the curative effect and adverse effects of moxibustion therapy for Polycystic Ovary Syndrome, in order to provide a reference for clinical treatment.
Condition being studied: Polycystic Ovary Syndrome (PCOS) is one of the most common endocrine diseases in women of adolescence and childbearing period. Its clinical manifestations include irregular menstruation, infertility, hyperandrogen signs, and polycystic ovary symptoms. Still, Patients with PCOS may also have 
metabolic abnormalities such as obesity, insulin resistance (IR), and dyslipidemia. PCOS is a high-risk factor for type 2 diabetes, cardiovascular and cerebrovascular diseases and endometrial cancer. At present, there is no effective cure for PCOS due to its unclear cause, therefore, symptomatic treatment has been the main treatment for a long time. Some recent trials indicate that moxibustion can be effective for PCOS. However, most of them are the combination of moxibustion and other therapies. To assess the effectiveness and safety of moxibustion treatment for PCOS, we designed this protocol for systematic review and metaanalysis.

\section{METHODS}

Participant or population: Women with Polycystic Ovarian Syndrome, regardless of race or educational and economic status.

Intervention: Moxibustion interventions will include needle warming moxibustion, thunder-fire moxibustion, mild moxibustion and so on.

Comparator: The controls can be placebo, other TCM interventions, pharmacotherapy, lifestyle management or blank control.

Study designs to be included: The review will include randomised controlled trials (RCT) with no language limitation.

Eligibility criteria: The review will only include randomised controlled trials of moxibustion treatment for women with PCOS. Crossover trials will not be included unless there first-phase data are available. NonRCTs reviews, case report, observational studies, animal experimental studies, expert experience, conference article and duplicated publications will be excluded.

Information sources: Electronic searches will be carried out up to November 2020 in the databases of The Cochrane Library, PubMed, MEDLINE, EMBASE, CNKI, WanFang, CBM and VIP. The International Prospective Register of Systematic
Reviews (PROSPERO), The Chinese Clinical Trial Registry Center and Clinical Trials was also searched for ongoing trials.

Main outcome(s): 1.Ovulation rate. 2.Monthly menstrual frequency. 3.Response rate.

Additional outcome(s): 1.Pregnancy rate. 2.Serum hormone levels (LH, LH/FSH ratio and Testosterone). 3.Insulin resistance level. 4.body mass index ( BMI ). 5.Adverse events.

Quality assessment / Risk of bias analysis: Two reviewers (Chen Wan and Kou Xu) will assess the methodological quality of all the random control trials based on the Cochrane Handbook 5.1.0 for Systematic Reviews of Interventions, which comprises selection bias(random sequence generation and allocation concealment), performance bias (blinding of participants and personnel), detection bias (blinding of outcome assessment), attrition bias (incomplete outcome data), reporting bias(selective reporting)and other bias. The studies will be evaluated as being of "low risk of bias," "high risk of bias," or "unclear risk of bias". At the same time, the included clinical trials will be awarded a score from 0 to 5 points according to Jadad scale evaluation criteria, which include reference to the generation of random sequences, blind enforcement, and withdrawal.

Strategy of data synthesis: We will synthesize and analyze the data by RevMan V.5.3(version 5.3 for windows; The Nordic Cochrane Centre, Copenhagen, Denmark). In the Cochrane Handbook, when heterogeneity was not significant $\left(I^{2} \leq 50 \%\right)$, a fixed effect model will be used to calculate the relative risk (RR) and weighted mean difference (WMD). when $I^{2} \geq 50 \%$, heterogeneity was regarded as statistically significant, and we will use a random-effect model to synthesize the data. The obvious clinical heterogeneity will be treated by the method of subgroup analysis or sensitivity analysis, or just descriptive analysis. 
Subgroup analysis: To make sure heterogeneity between included studies, subgroup analysis will be conducted only if there are sufficient number of articles in each subgroup. Criteria of subgroup analysis are as follows: 1.Different course of PCOS:Polycystic Ovary Syndrome of childbearing age, perimenopause and adolescence. 2.Different Types of moxibustion : direct moxibustion, indirect moxibustion, warm needling moxibustion, thunder-fire moxibustion, Du moxibustion, heat sensitive moxibustion and so on. 3.Different types of comparators, different types of population, different duration of intervention and different methodical quality of included studies.

Sensibility analysis: If there are sufficient studies, sensitivity analyses will be conducted to identify whether the following findings are robust. 1. Methodological qualities (e.g. random sequence generation, allocation concealment or participants/outcome assessment blinding). 2. Analysis-related issues (e.g. process of handling missing data).

Country(ies) involved: China.

Keywords: Polycystic Ovary Syndrome, PCOS and moxibustion, moxa and moxamoxibustion.

Contributions of each author:

Author 1 - Kou Xu - The author drafted the manuscript.

Email: 1932707307@stmail.hbtcm.edu.cn

Author 2 - Zhongyu Zhou - The second author carried out the research design.

Email: 2209447940@qq.com

Author 3 - Wei Huang.

Email: huangwei@hbhtcm.com

Author 4 - Siying Lv.

Email: 1321180818@qq.com

Author 5 - Yanji Zhang.

Email: zhangyanji1995@stmail.hbtcm.edu.cn

Author 6 - Chen Wan.

Email: 2014850922@qq.com 